\title{
A new design of portable intelligent detection equipment in chemical emergency rescue
}

\author{
Siwei Li, Yansong Liang, Yanran Feng \\ (Institute of NBC Defence, PLA Army, Beijing 102205)
}

\begin{abstract}
This paper proposed a portable intelligent mobile detection equipment aimed at emergency treatment of chemical accidents, and mainly introduces the function, features, structure design and key technology of the equipment. The function is realized mainly through internal real-time monitoring and leakage source location calculation. using this unmanned detection equipment can effectively reduce casualties and risks of emergency personnel, and make rescue work easier and safer.
\end{abstract}

\section{Introduction}

Recent years, leaks from chemical plants occur frequently, especially some of the toxic and noxious gases, can easily lead to human poisoning and casualties. Once it happened, timely and accurately finding and controlling of the source is the key to prevent the expansion and harm of the accident. Current source searching and controlling mainly rely on armed police or army actually entering the chemical plant for inspection. However, huge chemical field, large amounts of equipment, various gases, and unfamiliar with the topography of the chemical plant make it difficult to search the source and prevent it. Besides, when the accident occur, toxic fumes fill the chemical plant, oxygen levels are low, secondary leaks and explosions may occur at any time, which will cause physical harm or even life-threatening damage to emergency personnel.

In order to protect personnel's health and life safety, we developed a portable intelligent detection equipment for emergency rescue in chemical leak accidents.

\section{Function and feature}

The main functions of the equipment include the following aspects: (1)intelligent movement according to the preset motion trajectory or the self-planning path. (2) gas recognition and concentration measurement based on chemical detection system. (3) environmental scanning and three-dimensional map generation relying on a $360^{\circ}$ camera. (4) date monitoring and transmission in real time based on wireless communication technique. Through the collected concentration at each point, combined with the accident source algorithm and 3D map, we can finally determine the approximate position of the source, which is very helpful for the emergency personnel to enter into and perform the mission.

Features of the equipment are:
(1) autonomous intelligent detection with unmanned control.

(2) good ability to avoid obstacles and speed adjusted.

(3) multi-gas measured simultaneously with high accuracy and good sensitivity.

(4) panorama shot, day \& night conversion, and 3d map generation.

(5) real time monitoring and online analysis.

(6) the main technique is mature and system is stable.

(7) small size, simple structure, and good portability.

(8) higher performance cost ratio compared to current intelligent devices.

\section{Structure design}

The structure of the equipment mainly includes: intelligent mobile underpan, chemical detection module, environmental scanning module, and auxiliary detection module.






\subsection{Intelligent mobile underpan}

Currently, the ground detection robot is mainly tracked or wheeled. This type of intelligent robot has better ability to climb over obstacles and often used under bad outdoor conditions. For general emergency rescue in chemical plant leak accidents, a small size, portable, flexible, and economic detection device is more practical. Based on this requirement, we designed a new intelligent mobile equipment which integrates mechanism, electronics, sensor technology, automatic control, computer and so on. The underpan adopts a disc structure with a diameter of about $30 \mathrm{~cm}$. Two independent driving motors are installed on both sides, which realizes straight motion and rotation of the body with adjustable speed. The power supply adopts rechargeable battery, when it runs low, the underpan can automatically back to its charging base. In route designing, two algorithms are used as following: one is manually setting its motion and track, the other is self-planning and memory based on artificial intelligence system. The two ways can be switched freely to achieve the purpose of rapid mobile detection. Besides, considering the problem of avoiding obstacles on the move, we designed two sensing systems at the front end of the equipment.

\section{Infrared sensing system}

This system mainly uses the principle of infrared light induction, through infrared detection components, the received optical signal is converted to electrical signal for processing and analysis. Then the location of obstacle can be got which will help our equipment perceived environment.

$\checkmark \quad$ Bionic ultrasonic sensing system

The bionic ultrasonic sensing system uses sound waves similar to those emitted by dolphins and bats to detect obstacles, the process is to transmit pulse waves to the front and receive the returned, and calculate the time difference, according to the known sound velocity, we can get the position of obstacle.

\subsection{Chemical detection module}

In this module, gas sensor and adjusting circuit are used for recognition and concentration measurement. For different gas leak accidents, multiple gas sensors can be used in any combination freely to realize the detection of more than 30 common chemical gases such as carbon monoxide, nitric oxide, hydrogen sulfide, ammonia and so on.

Considering that electrochemical sensor is relatively mature and has low power consumption, high sensitivity and good anti-jamming capacity, we use it for the detection. Typical electrochemical sensor has three pins, working electrode, reference electrode, and counter electrode. The process is: target gas firstly diffuses into the sensor through inlet hole and passes the filter and

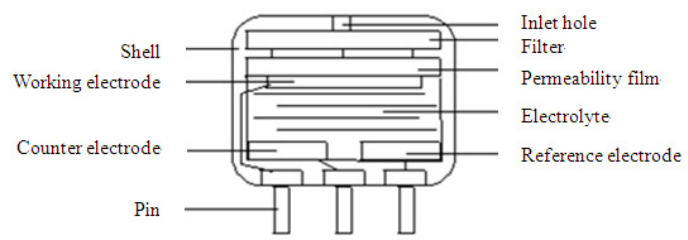

permeability film, then it reaches the surface of working electrode, and cause the redox reaction which lead to the circuit formation with counter electrode. Eventually, an electrical signal proportional to the gas concentration is generated.

The other important part is control circuit mainly composed of amplifying circuit, analog-digital conversion unit, and processer unit. Due to the weak current signal, the amplifier first enlarges it and then puts it into analog-digital conversion unit to convert, so that the signal can be analyzed and operated by the processor. The final data obtained will display on the equipment's screen and transmit wirelessly.

\subsection{Environmental scanning}

Another important function of the intelligent detection equipment is to scan the internal environment of the chemical plant. With this function module, the equipment can sense the environment better and get a faster and more accurate movement. In addition, the $3 \mathrm{~d}$ map generated would help emergency personnel reach the definite position. In this scanning module, we choose a 360-degree panoramic infrared camera, it is based on bionic fisheye structure, which makes space information in horizontal $360^{\circ}$ and vertical $180^{\circ}$ direction all imaged. Because of the wide filed-of-view characteristic, it is especially suitable for the large open spaces such as the chemical plant. In low light conditions, it can automatically switch to the infra-red mode which ensures the feasibility and clarity of night shooting. Besides, a microphone and loudspeaker is installed inside the camera which help us monitor the sound around so as to analyze whether there is a local explosion.

\subsection{Auxiliary detection module}

Auxiliary module mainly includes temperature sensor and pressure sensor to monitor the temperature and air pressure in the chemical plant.

\section{Wireless transmission technology}

Because wired networks are prone to irreversible damage in chemical accidents, which may cause communication disruption and affect the monitoring of internal environment, we choose the wireless transmission mode in this system. Current mainstream wireless technologies include: $3 \mathrm{G} / 4 \mathrm{G}$, Wi-Fi, Bluetooth, ZigBee and so on. Based on the consideration of transmission speed, distance, and cost, Wi-Fi is finally selected to serve the image transfer and data transfer module. Image transfer 
is to transmit the camera scanning to computer and build the $3 \mathrm{~d}$ map of chemical plant. Data transfer is to transmit the concentration, temperature, pressure collected to computer for next operation to analyze. Using the source inversion algorithm, combined with the data measured at each point, the approximate location of accident source can be got.

\section{Conclusion}

This paper proposed a new design of portable intelligent detection equipment used for chemical leak accident, which realized real-time monitoring of chemical plant and approximate source location detection. It is very important and significant for protecting the safety of emergency personnel. This equipment has a very broad application prospect due to its excellent performance, small size, good flexibility and portability, mainly suitable for the indoor rapid mobile detection.

\section{References}

1. Chen Longkai, South China University of Technology (2015)

2. Gong Pan, University of Science and Technology (2014)

3. Jia Guangli, Wei Juan, Ma Hongwei, Machine Tool \& Hydraulics, 36 (2008) 